\title{
The Association of Serum IL-10 Levels with the Disease Activity in Systemic-Onset Juvenile Idiopathic Arthritis Patients
}

\author{
Yu Peng $\mathbb{D}^{1,2}$ Xiaohui Liu, ${ }^{1,2}$ Zhao Duan, ${ }^{1,2}$ Junkai Duan $\mathbb{D}^{1,2}$ and Yulan Zhou $\mathbb{D}^{3}$ \\ ${ }^{1}$ Jiangxi Province Children's Hospital, Nanchang, 330006 Jiangxi, China \\ ${ }^{2}$ The Affiliated Children's Hospital of Nanchang University, Nanchang, 330006 Jiangxi, China \\ ${ }^{3}$ The First Affiliated Hospital of Nanchang University, Nanchang, 330006 Jiangxi, China \\ Correspondence should be addressed to Junkai Duan; yeduanjk@163.com and Yulan Zhou; wenxin_yl@163.com
}

Received 14 December 2020; Revised 1 February 2021; Accepted 10 March 2021; Published 22 March 2021

Academic Editor: Chiara De Luca

Copyright ( 2021 Yu Peng et al. This is an open access article distributed under the Creative Commons Attribution License, which permits unrestricted use, distribution, and reproduction in any medium, provided the original work is properly cited.

Objectives. Interleukin-10 (IL-10) has been suggested as a biomarker of disease activity in patients with adult-onset Still's disease (AOSD). In this study, we evaluated the serum IL-10 levels and investigated its clinical relevance in systemic-onset juvenile idiopathic arthritis (SoJIA). Methods. IL-10 levels were determined in 21 patients diagnosed with SoJIA and 35 patients with fever diseases which were suspected as SoJIA, and IL-10 levels were compared between SoJIA patients with regard to disease activity, disease courses, and other biomarkers. Results. Patients with SoJIA had significantly higher levels of IL-10 compared to patients with other febrile diseases. The serum levels of IL-10 were significantly higher in active SoJIA compared to inactive and positively correlated with known disease activity markers such as erythrocyte sedimentation rate (ESR), C-reactive protein level (CRP), ferritin (FER), and IL-6 levels. Moreover, the levels of IL-10 at diagnosis were significantly higher in SoJIA patients with a nonmonocyclic pattern than in patients with a monocyclic pattern. Compared to CRP, ESR, FER, and IL-6, IL-10 levels were superior in predicting monocyclic patients from nonmonocyclic patients. Conclusion. Compared to other febrile diseases, SoJIA patients have markedly higher levels of IL-10 which may assist with diagnosis. And a clear association of serum IL-10 levels with disease activity and disease courses in SoJIA was found. These results suggest that serum IL-10 might be a reliable clinical marker in SoJIA.

\section{Introduction}

Systemic-onset juvenile idiopathic arthritis (SoJIA) is a particular type of juvenile arthritis affecting children younger than 16 years of age, distinguished from the other subtypes of juvenile idiopathic arthritis (JIA) for its extra-articular features, such as spiking fever, evanescent rash, and serositis with elevated laboratory parameters of inflammation [1]. SoJIA is exceptional with its systemic features, which broaden the differential diagnosis to infections, malignancy, and Kawasaki disease [2]. Adult-onset Still's disease (AOSD), a systemic inflammatory disorder shared clinical similarities with SoJIA, is also known as the adult form of SoJIA [3]. Both of SoJIA and AOSD are considered to be multifactorial autoinflammatory diseases with inappropriate activation of the inflammatory cascade and disorder of inflammatory cytokine production [4]. Interleukin-10 (IL-10) is a pleomorphic cyto- kine with diverse phenotypic functions [5]. It is secreted by a wide variety of cells of the innate and adaptive immune system, including granulocytes, dendritic cells, macrophages, B cells, and $T$ cell subsets [6]. In the past several years, accumulated evidences have established an important role for IL-10 in many autoimmune and inflammatory diseases [7-9]. A recent study revealed that the serum concentrations of IL10 are increased in AOSD patients and positively correlated with disease activity [10]. It has been reported that the serum levels of IL-10 in SoJIA patients were changed in some studies $[11,12]$. However, these studies only compared the expression levels of IL-10 between SoJIA patients and healthy controls; little is known about the differences of IL-10 levels between SoJIA and other fever diseases. Furthermore, whether abnormal IL-10 production is also a hallmark of disease activity in SoJIA has not been examined in detail. In the present study, we measured the serum concentration of IL-10 
in patients with SoJIA and other diseases that can be easily misdiagnosed as SoJIA and analyzed the correlation between the serum levels of IL-10 and the clinical characteristics of SoJIA patients. We aimed to evaluate the utilization of serum IL-10 as a reliable clinical marker in SoJIA.

\section{Materials and Methods}

2.1. Patients. The serum levels of IL-10 in patients suspected or diagnosed with SoJIA have been measured before initiation of treatment as part of standard clinical care in the rheumatology department at Jiangxi Province Children's Hospital since 2016. In this noninterventional retrospective study, we retrospectively reviewed the data of 153 patients suspected or diagnosed with SoJIA that admission and follow-up at our department between January 2016 and December 2019. Medical histories and clinical and laboratory characteristics were collected from all subjects. Patients who had insufficient clinical or laboratory data were excluded. Complete data were available for 21 patients diagnosed with SoJIA, 7 patients diagnosed with systemic lupus erythematosus (SLE), 10 patients diagnosed with Kawasaki disease (KD), 6 patients diagnosed with acute lymphoblastic leukemia (ALL), and 12 patient diagnosed with severe infections (SIF). The demographic, clinical, and laboratory data for the patients are summarized in Table S1. For patients with clinically confirmed SoJIA, we also measured the serum levels of IL-10 during the follow-up, especially when disease activity changed. The therapy of hormones in combination with methotrexate (MTX) was as the basic program in patients with active disease. For some patients with severe disease manifestations, biologic agents or immunosuppressive agents are often added. In children with inactive disease, only nonsteroidal anti-inflammatory drugs were used to control SoJIA.

2.2. Diagnostic Criteria. The diagnosis of SoJIA was made according to the International League of Associations for Rheumatology (ILAR) Classification Criteria [13]. The diagnosis of SLE was performed in accordance with the standards developed by the American College of Rheumatology (ACR) [14]. KD was diagnosed based on the American Heart Association Guidelines [15]. The diagnosis of ALL was confirmed by a bone marrow biopsy. SIF were diagnosed by detection of pathogens in the blood, other body fluids, and tissue samples. Patients with SoJIA were further stratified into inactive SoJIA and active SoJIA. Disease activity status was assessed according to the clinical and laboratory data. The criteria for active disease were defined as follows: active arthritis, fever, rash, hepatosplenomegaly, generalized lymphadenopathy, and serositis, along with an elevated erythrocyte sedimentation rate (ESR) and C-reactive protein (CRP) levels [16]. Flare was defined as failure to control fever, a dramatic rise in inflammatory markers, worsening of other clinical features, or the progression of joint disease [17]. Inactive disease was defined according to American College of Rheumatology provisional criteria [18] as follows: no joints with active arthritis; no fever, rash, serositis, splenomegaly, or generalized lymphadenopathy attributable to JIA; no active uveitis to be defined; and ESR or CRP level within normal limits in the laboratory where tested.

2.3. Statistical Analysis. Descriptive data are presented as the mean \pm SD or median with an interquartile range (IQR) for continuous outcomes and number and percentage (\%) for categorical outcomes. Differences in data between multiple groups were analyzed using one-way ANOVA. Pairwise comparisons between groups were analyzed with Dunnett's T3 test. Differences between two groups were assessed by a 2-tailed $t$-test or Mann-Whitney $U$ test, as appropriate. The correlations between IL-10 and other variables of interest were evaluated with a Pearson correlation test or Spearman's correlation test. Receiver-operating characteristic (ROC) curve analysis was performed to assess the sensitivity and specificity of IL-10, CRP, ESR, FER, and IL-6 measurement and its diagnostic and predictive utility. In all comparisons, $p$ values less than 0.05 were considered statistically significant, and all $P$ values corresponded to two-sided significance tests. Statistical analyses were carried out using the SPSS software version 22.0.

\section{Results and Discussion}

3.1. Serum IL-10 Expression Levels. The demographic, clinical, and laboratory data for the patients are summarized in Table S1. There were some significant differences showed among the duration of fever before hospitalization, levels of serum IL-10, CRP, ESR, and FER in SoJIA compared with other febrile diseases like SLE, KD, ALL, and SIF. The serum concentration of IL-10 showed significant differences among various disease groups $(F=20.26, \quad P<0.001$, Figure 1(a)); the SoJIA group showed a significantly higher IL-10 expression level than the SLE group $(58.22 \pm 33.27$ vs. $\quad 28.20 \pm 16.90 \mathrm{pg} / \mathrm{mL}, \quad P<0.001), \quad \mathrm{KD}$ group $(28.97 \pm 20.12 \mathrm{pg} / \mathrm{mL}, P<0.001)$, ALL group $(18.72 \pm 10.25$ $\mathrm{pg} / \mathrm{mL}, P<0.001)$, and SIF group $(19.53 \pm 11.16 \mathrm{pg} / \mathrm{mL}, P$ $<0.001)$. Receiver of characteristic curve (ROC) analysis was conducted to analyze the performances of serum IL-10, CRP, ESR, and FER in supporting the diagnosis of SoJIA compared to the other fever diseases which were frequently misdiagnosed as SoJIA. Serum IL-10 performed well with area under the curve of (AUC) $0.862(P<0.001)$ in differentiating SoJIA from other disease categories (Figure 1(b)). This analysis indicated an optimal cut-off to identify SoJIA was $42.23 \mathrm{pg} / \mathrm{mL}$ with a sensitivity of $91.18 \%$ and a specificity of $66.67 \%$. The ACU of CRP, ESR, and FER are shown in Tables S2. Individual comparisons showed that serum IL-10 can effectively distinguish SoJIA from SLE, KD, ALL, or SIF. The cut-off values and their respective coordinates on the ROC curve are shown in Tables S3.

3.2. Serum IL-10 and Disease Activity. SoJIA patients were stratified by disease activity. The characteristics of the SoJIA patients with active disease (new onset or flare) or inactive disease are shown in Table 1 . We analyzed the association of serum IL-10 with disease activity in patients with SoJIA. As shown in Figure 2, serum IL-10 levels in patients with 

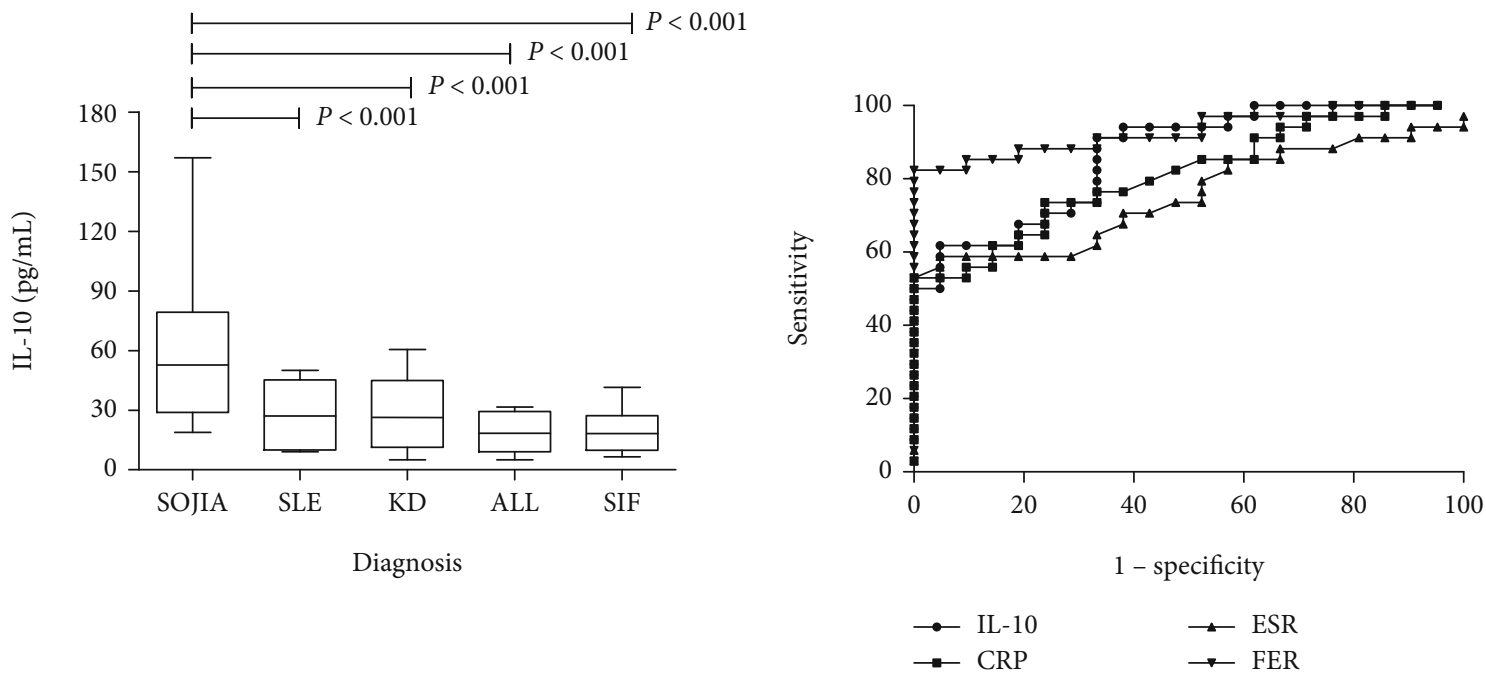

(a)

(b)

FIGURE 1: The expression of serum IL-10 and ROC curves. (a) Comparison of serum IL-10 levels in all patients. (b)ROC curve of IL-10, CRP, ESR, and FER in distinguishing SoJIA from other disease categories.

TABLE 1: Characteristics of the patients with active SoJIA or inactive disease.

\begin{tabular}{lccc}
\hline Characteristics & Inactive disease & New onset & Active disease \\
\hline Patients $(n)$ & 21 & 21 & 19 \\
Samples $(n)$ & 87 & 21 & 47 \\
IL-10 $(\mathrm{pg} / \mathrm{mL})$ & $18.33 \pm 9.10$ & $58.22 \pm 33.27$ & $64.49 \pm 26.55$ \\
CRP $(\mathrm{mg} / \mathrm{mL})$ & $3.78 \pm 1.87$ & $115.86 \pm 78.61$ & $73.64 \pm 29.51$ \\
ESR $(\mathrm{mm} / \mathrm{h})$ & $8.25 \pm 3.51$ & $71.14 \pm 19.99$ & $68.91 \pm 16.38$ \\
FER $(\mu \mathrm{g} / \mathrm{L})$ & $59.42 \pm 33.41$ & $2095.71 \pm 1281.48$ & $1881.64 \pm 999.12$ \\
IL-6 $(\mathrm{pg} / \mathrm{mL})$ & $53.96 \pm 96.81$ & $259.68 \pm 302.27$ & $197.35 \pm 115.38$ \\
\hline
\end{tabular}

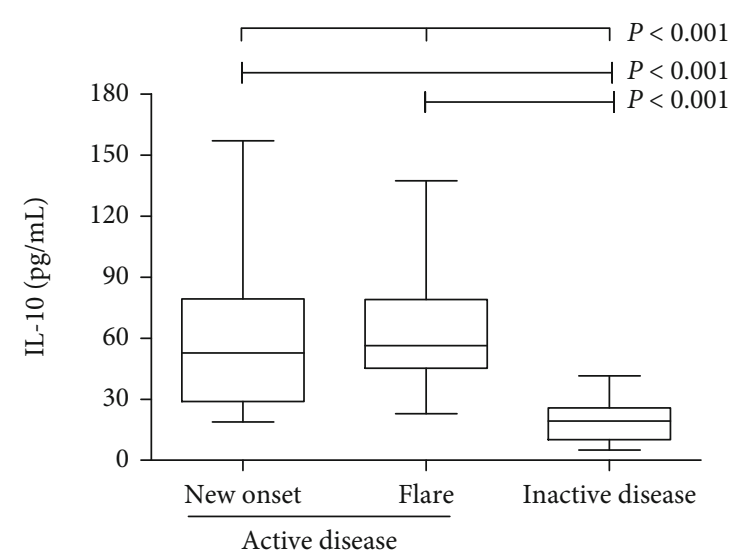

FIgURE 2: Comparison of serum IL-10 levels among patients with SoJIA with active and inactive disease activity.

SoJIA were higher during the active phase $(62.55 \pm 28.85$ $\mathrm{pg} / \mathrm{mL})$ than during the inactive phase $(18.33 \pm 9.10 \mathrm{pg} / \mathrm{mL}$, $P<0.001)$. But there was no significant difference in serum IL-10 levels between patients with new-onset SoJIA and patients with disease flares $(58.22 \pm 33.27$ vs. $64.49 \pm 26.55$ $\mathrm{pg} / \mathrm{mL} ; P=0.408)$.
3.3. Serum IL-10 and Inflammation Markers. An assessment of the correlation between the serum IL-10 levels and several known markers of disease activity was performed to further explore the relationship between serum IL-10 and disease activity in SoJIA. Our results revealed that the serum IL-10 levels correlated well with the levels of CRP, ESR, FER and IL-6 ( $r=0.624, P<0.001 ; r=0.748, P<0.001 ; r=0.664, P$ $<0.001$; and $r=0.377, P<0.001$; respectively) (Figure 3).

3.4. Serum IL-10 and Disease Courses. To assess the possible association between serum IL-10 and disease courses in patients with SoJIA, the SoJIA patients were divided into two groups. Patients who did not have a flare during the follow-up after initial remission were characterized as the monocyclic group. Patients who exhibited persistent systemic symptoms, or persistent arthritic symptoms, or both, and who experienced disease flares during the follow-up were characterized as the nonmonocyclic group. The characteristics of these two groups of patients are shown in Table 2. We analyzed the expression levels of serum IL-10 in these patients at diagnosis. Our results revealed that the serum IL10 levels at diagnosis were significantly higher in nonmonocyclic group patients than in monocyclic group patients 


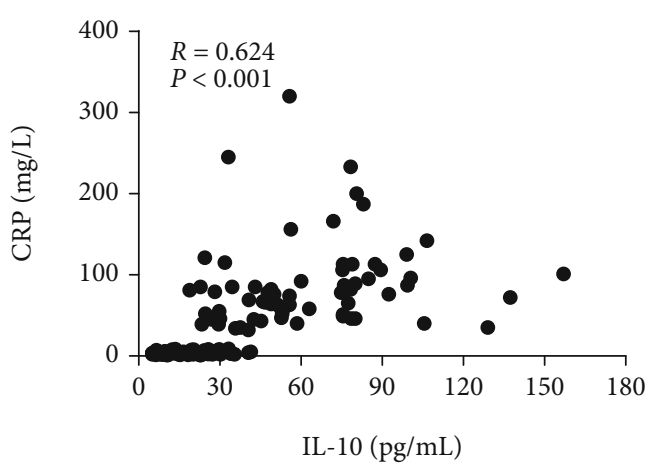

(a)

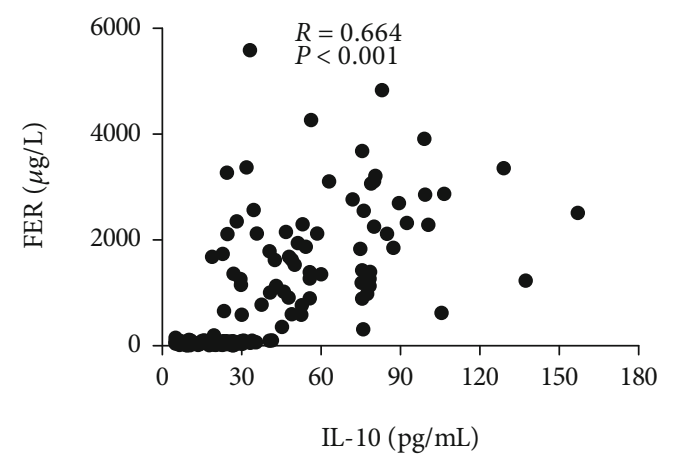

(c)

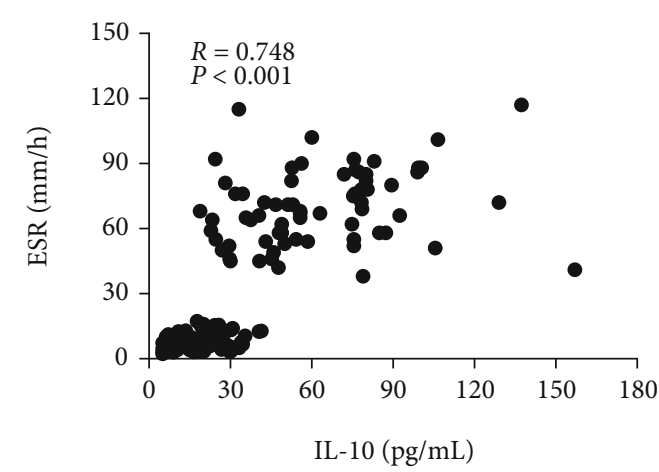

(b)

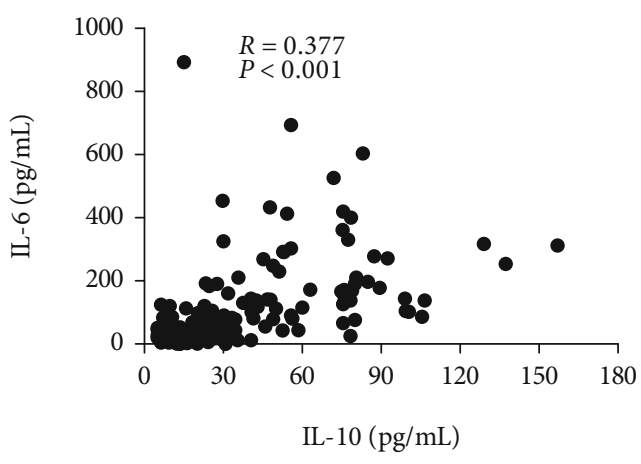

(d)

FIGURE 3: Correlation between serum IL-10 and other biomarkers. Serum IL-10 levels were positively correlated with CRP (a), ESR (b), FER (c), and IL-6 (d).

TABLE 2: Characteristics of SoJIA patients with monocyclic pattern or nonmonocyclic pattern.

\begin{tabular}{|c|c|c|c|c|}
\hline Characteristics & Monocyclic group & Nonmonocyclic group & $t$ & $P$ \\
\hline Patients $(n)$ & 12 & 9 & & \\
\hline IL-10 (pg/mL) & $41.61 \pm 18.14$ & $80.36 \pm 36.73$ & -3.190 & 0.005 \\
\hline CRP (mg/mL) & $94.00 \pm 60.12$ & $145.00 \pm 93.85$ & -1.518 & 0.145 \\
\hline $\operatorname{ESR}(\mathrm{mm} / \mathrm{h})$ & $67.00 \pm 16.85$ & $76.67 \pm 23.43$ & -1.102 & 0.284 \\
\hline $\operatorname{FER}(\mu \mathrm{g} / \mathrm{L})$ & $1712.25 \pm 654.62$ & $2607.00 \pm 1732.52$ & -1.473 & 0.172 \\
\hline IL-6 (pg/mL) & $116.69 \pm 59.63$ & $450.33 \pm 389.83$ & -2.545 & 0.034 \\
\hline
\end{tabular}

$(80.36 \pm 36.73 \quad$ vs. $\quad 41.61 \pm 18.14 \mathrm{pg} / \mathrm{mL}, \quad P=0.005)$ (Figure $4(\mathrm{a})$ ). To determine the predictive value of serum IL10 for disease courses, the ROC analysis was performed. The results demonstrated a better predictive accuracy for IL-10 than for CRP, ESR, FER, and IL-6 to detect a nonmonocyclic pattern. The ACU was 0.824 for IL-10, 0.667 for CRP, 0.664 for ESR, 0.648 for FER, and 0.810 for IL-6 (Figure 4(b)). At a cut-off value of $65.75 \mathrm{ng} / \mathrm{mL}$, serum IL-10 was $77.78 \%$ sensitive and $91.67 \%$ specific in differentiating between monocyclic patients and nonmonocyclic patients $(P=0.013)$ (Table S4).

\section{Discussion}

IL-10 is a pleiotropic cytokine produced by a wide variety of cells. It has been implicated in several major diseases including cancer, rheumatic disease, and infectious disease [5]. The levels of IL-10 are increased in many inflammatory diseases such as SLE, RA, and SSc. Recently, Sun et al. found that the serum concentrations of IL-10 are increased in AOSD patients and positively correlated with disease activity [10]. SoJIA shared clinical similarities with AOSD, despite the age of onset being younger than 16 years [19]; substantial advances have been made to confirm the homology between SoJIA and AOSD [20, 21]. However, the levels of IL-10 in SoJIA patients are still controversial. Cheng et al. revealed that the serum levels of IL-10 were increased in SoJIA patients compared with those in healthy controls [22]. Guo et al. found that the levels of IL-10 in children with SoJIA were lower than those in healthy children group [12]. Thus, to address this issue, we examined the serum levels of IL-10 in SoJIA patients with a highly sensitive electrochemiluminescence assay.

In this study, we compared the expression of serum IL-10 between SoJIA patients and other febrile diseases which were often misdiagnosed as SoJIA. The serum levels of IL-10 were significantly increased in SoJIA patients than in patients with 


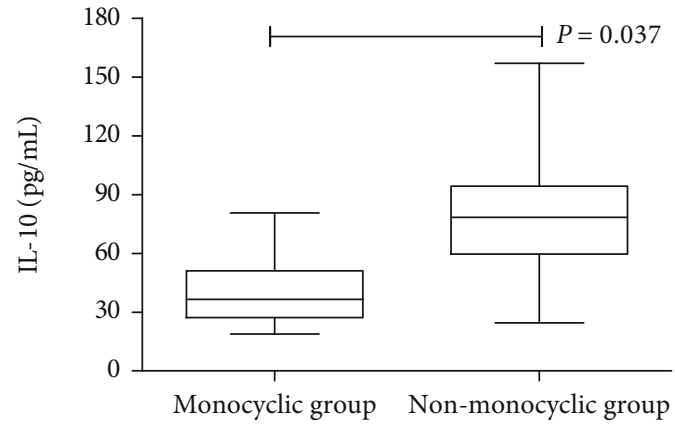

(a)

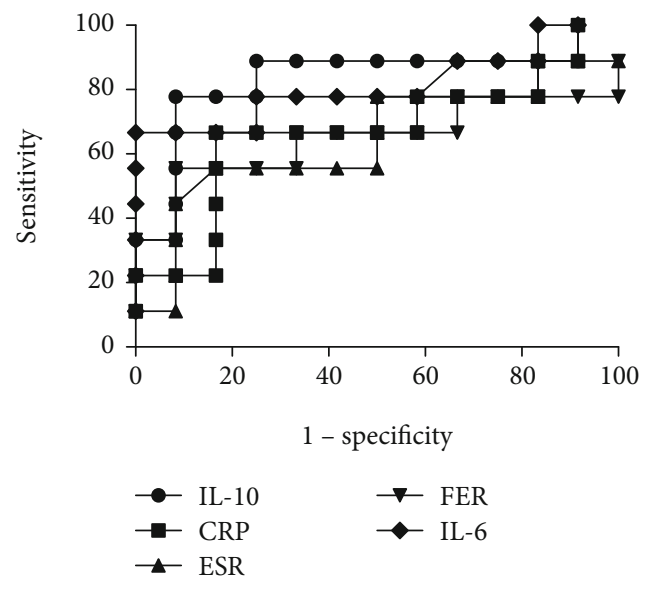

(b)

FIgURE 4: Correlation between serum IL-10 and disease courses. (a) Comparison of IL-10 levels among patients with SoJIA with monocyclic pattern and nonmonocyclic pattern. (b) ROC curves of IL-10, CRP, ESR, FER, and IL-6 for predicting disease course in SoJIA.

SLE, KD, ALL, or SIF. The diagnosis of SoJIA relies on clinical signs and symptoms. None of the specific biomarker had been established to date. Since many of the presenting symptoms are nonspecific, diagnosing SoJIA can be extremely challenging if typical arthritis is lacking. It is crucial to exclude other differential diagnoses such as infection, malignancy, other autoimmune disease, and autoinflammatory conditions [23]. Interestingly enough, we found that IL-10 would be a useful diagnostic marker for SoJIA. It can effectively distinguish SoJIA from other non-SoJIA causes of fever such as SLE, KD, ALL, or SIF. In addition, our results showed that the serum IL-10 levels in patients with SoJIA were higher during the active phase than during the inactive phase. Furthermore, serum IL-10 levels in SoJIA positively correlated with some nonspecific markers of inflammation such as ESR, CRP, FER, and a known disease activity marker IL-6 [23, 24]. Our results were similar with a study conducted in patients with AOSD, which found that the serum IL-10 was positively correlated with disease activity and inflammatory markers [10]. These findings strongly indicate that IL-10 has an important role in pathogenesis of SoJIA and can be a good marker for monitoring disease activity in SoJIA.

In addition to the diagnostic challenges associated with fevers of unknown origin and fever in children with SoJIA, prognostic challenges are prominent in SoJIA. SoJIA can be distinguished into three types according to the clinical courses: monocyclic, polycyclic, and persistent courses [25]. The monocyclic pattern, which is the most benign form, is characterized by a single illness episode attributed to SoJIA and then essentially disease remission. In the polycyclic pattern, the patient experiences recurrent attacks. Other patients exhibit a persistent pattern with unremitting disease manifested by persistent systemic, or persistent arthritic symptoms, or both [26]. The prognosis of SoJIA is largely dependent upon the disease course. Long-term outcome studies have indicated that patients with SoJIA with polycyclic or persistent disease course display greater disability, weakness, and disease damage than those with monocyclic disease course [2]. Therefore, detecting disease course in its early stage may be essential to the management of SoJIA. However, guidance on establishing an early detection of disease course in SoJIA is limited. Recently, Zhao et al. found that early production of IL-10 was significantly associated with disease severity in COVID-19, a global and life-threatening pandemic which is characterized by high levels of cytokines such as IL-1, IL-2, IL-4, IL-6, and IL-10 in infected individuals $[27,28]$. Furthermore, Han et al. found that IL-6 and IL-10 can be used as predictors for fast diagnosis of COVID-19 patients with higher risk of disease deterioration [29].

Our data show that the serum IL-10 levels at diagnosis were significantly higher in SoJIA patients with polycyclic or persistent disease course than those patients with monocyclic disease course. Further, with our present results, we reveal a better predictive accuracy for IL-10 than for CRP, ESR, FER, and IL- 6 to detect a nonmonocyclic pattern, which means that IL-10 can be used to discriminate SoJIA patients at diagnosis as prone to a monocyclic disease course or other disease courses. The additional ability to detect disease courses with a higher sensitivity than ESR, FER, and IL-6 makes the assessment of serum IL-10 levels a valuable tool in the clinical assessment of patients with SoJIA.

It is well known that IL-10 mainly acts as an antiinflammatory cytokine in many diseases [5]. Interestingly, our data confirmed the increased serum levels of IL-10 in patients with SoJIA and demonstrated a possible role of IL10 as a disease biomarker in SoJIA. A recent study revealed that serum levels of IL-37, which functions as a natural suppressor of inflammatory and immune responses, were increased in patients with AOSD and associated with AOSD disease activity [30]. Moreover, their data show that serum IL-37 levels were positively correlated with IL-10. They speculate that inflammation signaling not only exacerbated the inflammatory response in the pathogenesis of AOSD but also promoted the expression of anti-inflammatory cytokines such as IL-37 and IL-10 to limit excessive inflammation in AOSD $[30,31]$. According to the above results, we hypothesize that there exists a similar principle in IL-10 and a feedback loop from proinflammatory cytokines to the upregulation of anti-inflammatory cytokines in SoJIA. 
This study has several limitations. Small sample was the first limitation due to rarity of SoJIA. Another limitation was the insufficiency of follow-up time. As the definition of disease course is strongly based on the follow-up time, we would not know if some of the monophasic patients might flare in the future, although all monophasic patients were followed at least several years in our center without any flare. Then, the long-term outcomes of patients might be different from the visit in our center in terms of disease courses. Finally, it is a single-center, retrospective cohort study. Therefore, multicenter studies with larger cohorts will be necessary to further verify our findings.

To summarize, our study showed that upregulation of IL10 was positively correlated with SoJIA disease activity, and the levels of IL-10 at diagnosis might predict the disease courses of SoJIA. Monitoring of serum IL-10 levels may be useful for assessing SoJIA disease activity and predicting SoJIA disease courses, although further large studies with a greater number of SoJIA patients are necessary to confirm the usefulness of serum IL-10 in daily clinical practice.

\section{Data Availability}

Data was available on request.

\section{Conflicts of Interest}

The authors declare that they have no conflicts of interest.

\section{Authors' Contributions}

YP performed statistical analysis and wrote the first draft of the manuscript. YP, XL, and ZD collected clinical information. $\mathrm{YZ}$ and JD designed the study and helped to revise the manuscript. All authors read and approved the final manuscript.

\section{Acknowledgments}

This work was supported by the National Key Research and Development Program of Jiangxi Province (20202BBGL73024) and National Natural Science Foundation of Jiangxi Province (20202BAB216011). We thank all of the patients and their families for their participation in this research.

\section{Supplementary Materials}

Table S1: clinical characteristics of patients with SoJIA and other fever diseases. Table S2: analysis of the ROC curves of IL-10, CRP, ESR, and FER in diagnosing SoJIA. Table S3: analysis of the ROC curves of IL-10. Table S4: analysis of the ROC curves of IL-10, CRP, ESR, FER, and IL-6 in predicting disease course. (Supplementary Materials)

\section{References}

[1] R. Cimaz, "Systemic-onset juvenile idiopathic arthritis," Autoimmunity Reviews, vol. 15, no. 9, pp. 931-934, 2016.

[2] H. E. Baris, E. Anderson, B. Sozeri, and F. Dedeoglu, "Impact of biologics on disease course in systemic onset juvenile idio- pathic arthritis," Clinical Rheumatology, vol. 37, no. 12, pp. 3263-3273, 2018.

[3] R. Giacomelli, P. Ruscitti, and Y. Shoenfeld, "A comprehensive review on adult onset Still's disease," Journal of Autoimmunity, vol. 93, pp. 24-36, 2018.

[4] K. Theodoropoulou and S. Georgin-Lavialle, "Systemic juvenile onset idiopathic arthritis and adult onset still disease," Revue Medicale Suisse, vol. 14, no. 594, pp. 372-377, 2018.

[5] C. Neumann, A. Scheffold, and S. Rutz, "Functions and regulation of T cell-derived interleukin-10," Seminars in Immunology, vol. 44, p. 101344, 2019.

[6] H. Wei, B. Li, A. Sun, and F. Guo, "Interleukin-10 family cytokines immunobiology and structure," Advances in Experimental Medicine and Biology, vol. 1172, pp. 79-96, 2019.

[7] S. Kany, J. T. Vollrath, and B. Relja, "Cytokines in inflammatory disease," International Journal of Molecular Sciences, vol. 20, no. 23, p. 6008, 2019.

[8] W. Ouyang, S. Rutz, N. K. Crellin, P. A. Valdez, and S. G. Hymowitz, "Regulation and functions of the IL-10 family of cytokines in inflammation and disease," Annual Review of Immunology, vol. 29, no. 1, pp. 71-109, 2011.

[9] X. Wang, K. Wong, W. Ouyang, and S. Rutz, "Targeting IL-10 family cytokines for the treatment of human diseases," Cold Spring Harbor Perspectives in Biology, vol. 11, no. 2, 2019.

[10] Y. Sun, Z. Wang, H. Chi et al., "Elevated serum levels of interleukin-10 in adult-onset Still's disease are associated with disease activity," Clinical Rheumatology, vol. 38, no. 11, pp. 3205-3210, 2019.

[11] A. A. Shahin, O. G. Shaker, N. Kamal, H. A. Hafez, W. Gaber, and H. A. Shahin, "Circulating interleukin-6, soluble interleukin-2 receptors, tumor necrosis factor alpha, and interleukin-10 levels in juvenile chronic arthritis: correlations with soft tissue vascularity assessed by power Doppler sonography," Rheumatology International, vol. 22, no. 2, pp. 8488, 2002.

[12] L. Guo, M.-P. Lu, Y.-M. Tang et al., "Serum cytokine levels in children with newly diagnosed active systemic juvenile idiopathic arthritis," Chinese journal of contemporary pediatrics, vol. 16, no. 12, pp. 1241-1244, 2014.

[13] R. E. Petty, T. R. Southwood, P. Manners et al., "International League of Associations for Rheumatology classification of juvenile idiopathic arthritis: second revision, Edmonton, 2001," Journal Rheumatology, vol. 31, no. 2, pp. 390-392, 2004.

[14] M. C. Hochberg, "Updating the American College of Rheumatology revised criteria for the classification of systemic lupus erythematosus," Arthritis \& Rheumatism, vol. 40, no. 9, p. $1725,1997$.

[15] B. W. McCrindle, A. H. Rowley, J. W. Newburger et al., "Diagnosis, treatment, and long-term management of Kawasaki disease: a scientific statement for health professionals from the American Heart Association," Circulation, vol. 135, no. 17, pp. e927-e999, 2017.

[16] M. Mizuta, M. Shimizu, N. Inoue, Y. Nakagishi, and A. Yachie, "Clinical significance of serum CXCL9 levels as a biomarker for systemic juvenile idiopathic arthritis associated macrophage activation syndrome," Cytokine, vol. 119, pp. 182-187, 2019.

[17] T. J. A. Lehman, Systemic-Onset Juvenile Idiopathic Arthritis, in: Sawhney, A. Aggarwal, Ed., Singapore, Pediatric Rheumatology, Springer Science and Business Media, 2017. 
[18] C. A. Wallace, E. H. Giannini, B. Huang et al., “American College of Rheumatology provisional criteria for defining clinical inactive disease in select categories of juvenile idiopathic arthritis," Arthritis Care Res (Hoboken), vol. 63, no. 7, pp. 929-936, 2011.

[19] E. Feist, S. Mitrovic, and B. Fautrel, "Mechanisms, biomarkers and targets for adult-onset Still's disease," Nature Reviews Rheumatology, vol. 14, no. 10, pp. 603-618, 2018.

[20] N. Inoue, M. Shimizu, S. Tsunoda, M. Kawano, M. Matsumura, and A. Yachie, "Cytokine profile in adultonset Still's disease: Comparison with systemic juvenile idiopathic arthritis," Clinical Immunology, vol. 169, pp. 8-13, 2016.

[21] Y. Jamilloux, S. Georgin-Lavialle, P. Seve, A. Belot, and B. Fautrel, "It is time to reconcile systemic juvenile idiopathic arthritis and adult-onset Still's disease," La Revue de Médecine Interne, vol. 40, no. 10, pp. 635-636, 2019.

[22] J. Hirao, S. Hibi, T. Andoh, and T. Ichimura, "High levels of circulating lnterleukin- 4 and lnterleukin-10 in Kawasaki disease," International Archives of Allergy and Immunology, vol. 112, no. 2, pp. 152-156, 2004.

[23] J. J. Y. Lee and R. Schneider, "Systemic Juvenile Idiopathic Arthritis," Pediatric Clinics of North America, vol. 65, no. 4, pp. 691-709, 2018.

[24] S. Vilaiyuk, B. Lerkvaleekul, S. Soponkanaporn, C. Setthaudom, and S. Buranapraditkun, "Correlations between serum interleukin 6 , serum soluble interleukin 6 receptor, and disease activity in systemic juvenile idiopathic arthritis patients treated with or without tocilizumab," Central European Journal of Immunology, vol. 44, no. 2, pp. 150-158, 2019.

[25] N. Bruck, A. Schnabel, and C. M. Hedrich, "Current understanding of the pathophysiology of systemic juvenile idiopathic arthritis (sJIA) and target-directed therapeutic approaches," Clinical Immunology, vol. 159, no. 1, pp. 72-83, 2015.

[26] P. Efthimiou and S. Yadlapati, “Adult-Onset Still's Disease," in Auto-Inflammatory Syndromes, pp. 261-276, Springer, Cham, 2018.

[27] Y. Zhao, L. Qin, P. Zhang et al., "Longitudinal COVID-19 profiling associates IL-1RA and IL-10 with disease severity and RANTES with mild disease," JCI Insight, vol. 5, no. 13, 2020.

[28] S. Dhar, K. V. Priyan, S. Damodar, S. Gujar, and M. Das, "IL-6 and IL-10 as predictors of disease severity in COVID-19 patients: results from meta-analysis and regression," Heliyon, vol. 7, no. 2, 2021.

[29] H. Han, Q. Ma, C. Li et al., "Profiling serum cytokines in COVID-19 patients reveals IL- 6 and IL-10 are disease severity predictors," Emerging Microbes \& Infections, vol. 9, no. 1, pp. 1123-1130, 2020.

[30] H. Chi, D. Liu, Y. Sun et al., "Interleukin-37 is increased in adult-onset Still's disease and associated with disease activity," Arthritis Research \& Therapy, vol. 20, no. 1, p. 54, 2018.

[31] G. Cavalli and C. A. Dinarello, "Suppression of inflammation and acquired immunity by IL-37," Immunological Reviews, vol. 281, no. 1, pp. 179-190, 2018. 